\title{
Magnon Excitations in Manganites
}

\author{
P. Aleshkevych*, M. Baran and H. Szymczak \\ Institute of Physics, Polish Academy of Sciences \\ al. Lotników 32/46, 02-668 Warsaw, Poland
}

\begin{abstract}
A review of experimental studies of magnon excitations in manganites is presented. Two kinds of techniques: the inelastic neutron scattering (in bulk materials) and the microwave resonance (in thin films) are considered. Experimental studies of spin dynamics by inelastic neutron scattering in metallic ferromagnetic manganites have shown that at low temperature for small wave vectors $k \rightarrow 0$ the dispersion relation has a quadratic shape similar to that observed in Heisenberg ferromagnets. However, the above technique although very informative can be used only for sufficiently large samples of bulk materials. A complementary microwave resonance technique allows studying not only bulk properties, but also surface properties. There are two main theoretical approaches used to interpret the spin wave resonance spectra: the volume inhomogeneity and the surface inhomogeneity models. The last one introduced by Puszkarski has allowed for interpretation of the observed surface magnon excitations in thin films.
\end{abstract}

PACS numbers: 75.30.Ds, 75.47.Lx, 75.70.-i, 76.50.+g

\section{Introduction}

Manganites are known due to their unique magnetic and electronic properties resulting from competing charge, exchange, and phonon interactions [1]. The behavior of these compounds, including their colossal magnetoresistance, is determined by their atomic order and the presence of compositional or structural inhomogeneities [2-4]. Their properties can suggest a great perspective for practical applications [5-7]. The thin films are often required for practical applications. New possibilities of preparing high-quality single crystalline epitaxial thin films have caused a revival of interest to manganites [8].

The understanding of the nature of spin wave excitations and their relation with the magnetic structure, charge ordering, and orbital ordering is essential for

*corresponding author; e-mail: pavloa@ifpan.edu.pl 
a comprehensive description of magnetism, transport, and colossal magnetoresistance in manganites. The magnon excitations spectrum is a unique source of the information answering on such fundamental question as the proper choice of effective Hamiltonian that would confirm of appropriate theoretical model. There are numerous studies concerning magnetic excitations in bulk samples of manganites while only few works explore spin waves in thin films of manganites. It is a purpose of this paper to review experimental works concerning manganites from the point of view of magnon excitations and to compare results for bulk and thin film samples of manganites.

In general, there are two types of magnetic excitations in ferromagnets:

(i) Stoner excitations, in which an electron is excited from an occupied state of the majority spin channel to an empty state of minority spin channel creating an electron-hole pair of triplet spin. These excitations are responsible for longitudinal fluctuations of the magnetization.

(ii) Magnons or spin waves, responsible for collective transverse fluctuations of the magnetization. In insulators the spin waves are well defined throughout the Brillouin zone and may be described by Heisenberg model. In metals, spin waves disappear for the certain wave vector $k$ at entering into the Stoner excitations zone.

The splitting separating these two spectra $\left(E_{0}\right)$ is deciding which types of excitations are prevailing. In manganites both the localized $\mathrm{Mn} t_{2 \mathrm{~g}}$ electrons and the delocalized $\mathrm{Mn} e_{\mathrm{g}}$ electrons reside at the same site and their spins are coupled ferromagnetically by a strong on-site Hund coupling $J_{\mathrm{H}}$. At the lowest temperatures, $E_{0} \approx 2 J_{\mathrm{H}}$ is about $2 \ldots 4 \mathrm{eV}$ [9] and since the typical magnon bandwidth is about $0.1 \mathrm{eV}[10]$, the spin wave excitations are well separated from the Stoner excitations. Therefore, a reasonable approximation is to neglect completely the Stoner excitations and to focus only on the spin waves.

\section{Inelastic neutron scattering}

The most effective experimental method for studying magnetic excitations is the inelastic neutron scattering (INS). There are numerous works [10-25] where INS was used to systematic investigations of the spin-wave excitations (magnons) in various manganites. Stoichiometric $\mathrm{LaMnO}_{3}$ is one of the first manganites where this technique was used [11]. Results were described by the effective Hamiltonian taking into account the isotropic Heisenberg interaction and one-ion anisotropy, from which it was determined that an exchange interaction is ferromagnetic in the $(a b)$ plane and antiferromagnetic along the $c$ axis. $\mathrm{La}_{0.7} \mathrm{~Pb}_{0.3} \mathrm{MnO}_{3}$ is the first of ferromagnetic manganites whose dispersion was found throughout the entire Brillouin zone [10]. The results revealed that the effective spin-Hamiltonian is the same as for a simple cubic Heisenberg ferromagnet with a single nearest neighbor coupling. In this case the dispersion relation is given by a following simple relation: 


$$
E=\Delta+\left(E_{\mathrm{SW}} / 6\right)\left[3-\cos \left(k_{x} a_{0}\right)-\cos \left(k_{y} a_{0}\right)-\cos \left(k_{z} a_{0}\right)\right],
$$

where $\Delta$ represents the spin wave energy gap between the ground state and the state with all spins parallel; $E_{\mathrm{SW}}$ is a magnon bandwidth. A numerous investigation confirmed that in all metallic ferromagnetic manganites, their low-temperature magnetic excitations are conventional spin waves with very similar dispersions at long wavelength given by

$$
E=\Delta+D(T) k^{2},
$$

where $\Delta$ represents the spin waves energy gap, and the spin stiffness coefficient $D(T)$ is directly related to the exchange interactions. While in early works $\Delta$ have been found to be nonzero from a dispersion fitting $[9,10]$, the later investigations show that $\Delta$ is too small $\left(\sim 1 \times 10^{-5} \mathrm{eV}\right)$ to be measured directly with the INS technique in ferromagnetic regime [26].

What is specific for magnon excitations in manganites, these are numerous evidences of a departure from the simple local moments model: it is softening or flattering of the spin waves dispersion for large $k$ close to the edge of the Brillouin zone, as well as splitting of the dispersion relation for intermediate $k$. It was suggested in [23] that this spin wave softening as well as damping are generic to the double exchange (DE) ferromagnet including those with large $T_{\mathrm{C}}$. There are different explanations of dispersion departure for large $k$ in literature. In the first one, an attempt was presented to take into account the exchange interaction with spins from 2,3,4-th coordination spheres apart from the interactions between nearest-neighbors [18]. There are also theoretical [27] as well as experimental [28] results pointing that orbital ordering leads to softening and damping of the zero-boundary magnons. However, at the present time, the opinion dominates that a magnon-phonon interaction is a major source responsible for "softening" spin waves for large $k$. Thermal fluctuations of magnetic ions lead to decreasing exchange interactions in turn weakening spin waves with energies higher than appropriate phonon ones $[21,29,30]$. Also one of possible consequences of such magnon-phonon interaction is to create energy gaps in the magnon dispersion at the nominal intersections of the magnon and phonon modes [16, 21].

The strong damping of spin waves even at the lowest temperatures $[16,18]$ is also peculiar for manganites in contrast to conventional ferromagnets. In the past, it was assumed that the magnon dispersion enters the Stoner continuum at finite $k$ and $\omega$, where the quasi-particle damping of spin waves occurs. At present, it is generally accepted that the strong damping and its directionality is reminiscent of the $3 d$ itinerant electron ferromagnets [29].

\section{Microwave technique}

The inelastic neutron scattering is a powerful technique in studying the spin dynamics in large samples; however, it experiences some difficulties when thin films 
are necessary to study. Fortunately, the microwave technique is a very valuable method for studying magnon excitations in thin films. The microwave field may excite spin waves in ferromagnets. The important problem concerning the microwave technique is an achievement of film's specific boundary conditions necessary for the spin-wave resonance (SWR) appearance. The next important problem is the proper choice of the appropriate theoretical model for the data interpretation. The majority of existing models concerning SWR could be divided into the two main types: the volume inhomogeneity (VI) model and surface inhomogeneities (SI) one. The essential difference between those models relies on a formulation of the boundary conditions in the film. These conditions are leading to pinning of surface spins. The VI model assumes the volume magnetization maximal in the middle of film and decreasing towards surfaces providing pinned surface spins [31]. In the SI model [32-35], a volume magnetization is assumed to be homogeneous and a surface anisotropy is responsible for the pinning of the surface spins. A special interest in SWR consists in the fact that additional resonance lines corresponding to surface spin waves could be observed [35-38]. An existence of acoustic surface spin waves in the ferromagnet implies that its ground state is not uniform.

Thus far, there are only few experimental works where the spin wave resonance was observed in manganites [39-43]. The SWR in manganites was observed, for the first time, by Lofland et al. [39] in $\mathrm{La}_{0.67} \mathrm{Ba}_{0.33} \mathrm{MnO}_{3}$ thin films and next by Yin et al. [40] in $\mathrm{La}_{0.75} \mathrm{Sr}_{0.11} \mathrm{Ca}_{0.14} \mathrm{MnO}_{3}$ thin films. In both cases the authors have used the ideal Kittel model to describe their results, moreover for $\mathrm{La}_{0.67} \mathrm{Ba}_{0.33} \mathrm{MnO}_{3}$ the temperature dependence of the spin-wave stiffness coefficient was evaluated and found to be in agreement with the spin-wave theory. The last known case was $\mathrm{La}_{0.7} \mathrm{Mn}_{1.3} \mathrm{O}_{3-\delta}$ films, where the microwave technique

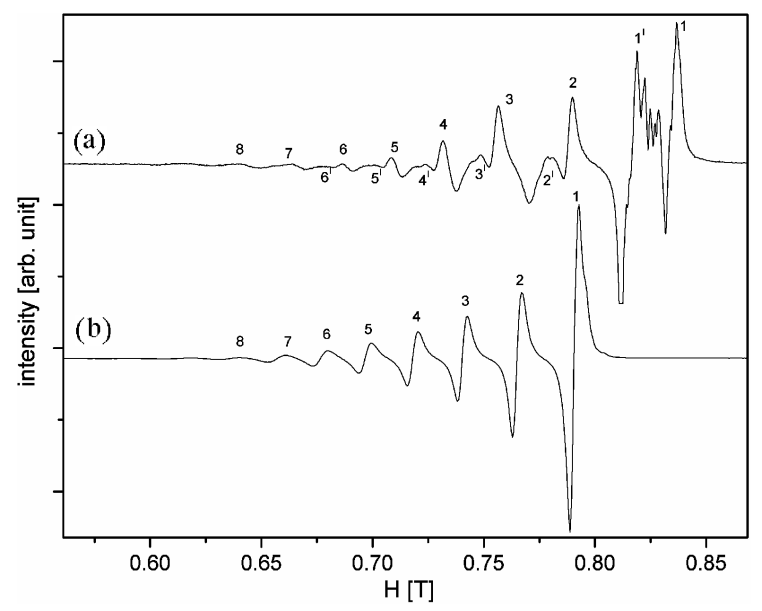

Fig. 1. Two resonance spectra recorded at $T=165 \mathrm{~K}$ in perpendicular orientation. The first one has been recorded a long time after a post preparation annealing (a), the next one - immediately after the second annealing in the same conditions (b). 
was used to excite spin waves successfully [41-43]. The results, unpublished thus far, for these films will be described here in details.

The $\mathrm{La}_{0.7} \mathrm{Mn}_{1.3} \mathrm{O}_{3-\delta}$ films preparation has been described elsewhere [44, 45]. The SWR experiments have been carried out using the X-band spectrometer with a reflection cavity, operating at a fixed frequency of about $9.25 \mathrm{GHz}$ and equipped with a variable temperature flowing gas cryostat.

It was found that the spectrum reveals a complex structure at temperatures below the Curie temperature. An important feature of the spectra is the presence of resonance absorption from surface waves at low temperatures [42, 43]. A very unusual transformation of the SWR spectrum comes when the temperature becomes higher than $150 \mathrm{~K}$. In this case each of the resonance lines begins to be split into two lines. This splitting grows with the increase in temperature. A typical example of such spectrum recorded at $165 \mathrm{~K}$ is shown in Fig. 1 as (a). That "doublet structure" of the SWR spectrum can be easily explained in frames of the SI model [36]. The values of wave vectors (that determine resonance peaks positions) can be found from the following characteristic equation:

$$
\operatorname{tg}(L k)=\frac{\left(A_{\mathrm{f}} A_{\mathrm{S}}-1\right) \sin (k a)}{\left(A_{\mathrm{f}} A_{\mathrm{S}}+1\right) \cos (k a)-\left(A_{\mathrm{f}}+A_{\mathrm{S}}\right)},
$$

where $L$ - thickness of a film, $k$ - wave vector, $a$ - lattice constant, $A_{\mathrm{f}}$ and $A_{\mathrm{S}}$ - surface parameters for free surface and interface, respectively. Only two parameters $\left(A_{\mathrm{f}}, A_{\mathrm{S}}\right)$ define a set of wave vectors - roots of $(3)$. Upon changing some of these parameters, Eq. (3) should give another set of wave vectors not conterminous with the previous set. It means, that the doublet structure of the SWR spectrum could be explained, if a film would be characterized by three different surface parameters (which would require that one of surfaces should be characterized by two surface parameters). A description of a surface by two parameters is possible in the case if magnetic inhomogeneities are created on that surface with a periodical topology [46]. In the $\mathrm{La}_{0.7} \mathrm{Mn}_{1.3} \mathrm{O}_{3-\delta}$ manganites (as well as in any other manganites), the existence of two types of manganese ions: $\mathrm{Mn}^{3+}$ and $\mathrm{Mn}^{4+}$ could be a natural source of that magnetic inhomogeneity. However, those ions should be aligned periodically, forming two magnetic sublattices (or stripes). It is important to note that even at the presence of two types of ions but without a periodic structure, such surface would be characterized only by one average value of surface parameter. Such periodic stripe structure was observed in manganites $\mathrm{La}_{1-x} \mathrm{Ca}_{x} \mathrm{MnO}_{3}(x>0.5)$ using the electron microscopy technique [47, 48]. But manganites studied in these papers are charge-ordered antiferromagnets in contrast to our samples, which are ferromagnetically ordered. Nevertheless, several theoretical and experimental investigations indicated the existence of charge-ordered phase also in ferromagnets [49-51].

A simple way to confirm an assumption of stripe formation by the Mn ions would change the ratio $\mathrm{Mn}^{3+} / \mathrm{Mn}^{4+}$ on the free surface, leading to its better 
homogeneity. This could be done by the film annealing in oxygen atmosphere. That second annealing was carried on in the same conditions as the first post-preparation annealing. As it was expected, the repeated annealing has not changed magnetic properties in the whole volume of the film, since the value of the magnetic moment has remained the same as it was before, but this operation has changed strongly the free surface properties. Figure 1 illustrates how the new annealing influenced the SWR spectrum. The spectrum (a) has been recorded a long time after post preparation annealing, whereas the spectrum (b) - immediately after the second annealing. As one can see the spectrum (b), recorded just after additional annealing, does not show any traces of splitting. At annealing the surface absorbs oxygen, therefore, the part of $\mathrm{Mn}^{3+}$ ions is transformed into $\mathrm{Mn}^{4+}$. The reduced ratio $\mathrm{Mn}^{4+} / \mathrm{Mn}^{3+}$ makes a free surface magnetic structure homogeneous that results in disappearance of splitting in the spectrum.

The multipeak resonance spectrum allows one to check the dispersion relation since a position of each resonance peaks is proportional to the energy of an appropriate spin wave according to the well-known Kittel resonance condition [32]:

$$
\frac{\omega}{\gamma}=H_{\mathrm{res}}-4 \pi M+H_{\mathrm{a}}+D k^{2},
$$

where $H_{\text {res }}$ is the resonance field, $\omega$ - resonance frequency, $M$ - saturation magnetization, $H_{\mathrm{a}}$ - anisotropy field, and $\gamma$ is the spectroscopic splitting ratio. As it follows from (5), the dependence of $H_{\text {res }}$ as a function of $k^{2}$ should be linear. The dependence $H_{\text {res }}\left(k^{2}\right)$ for a spectrum recorded at three different temperatures in the perpendicular orientation is shown in the inset of Fig. 2. As it can be remarked, for the most long-wave modes there is a deviation from a simple linear dependence. It is related to the fact that these modes (and especially surface modes) are more sensitive to an inhomogeneity of magnetic structure in a surface layer. Therefore, to determine a more realistic value of $D$ it is necessary to take into account only the linear part of dispersion dependence. In this case $D$ will hold information about a bulk average exchange constant. In Fig. 2 the temperature dependence of $D$ is shown. From these data, a value of $D(0)=156 \mathrm{meV} \cdot \AA^{2}$ was found. This value agrees within the experimental error with the one determined from the magnetization saturation temperature dependence [43].

For spin waves with small $k$ the experimental $D(T)$ data can be compared with the Dyson formalism of two-spin-wave interactions in a Heisenberg ferromagnet [52], which predicts that the dynamical interaction between the spin waves gives a $T^{5 / 2}$ behavior:

$$
D(T)=D(0)\left\{1-\frac{v_{0} \bar{l}^{2} \pi}{S} \zeta(5 / 2)\left[\frac{k_{\mathrm{B}} T}{4 \pi D(0)}\right]^{5 / 2}+\ldots\right\},
$$

where $v_{0}$ is the volume of the unit cell determined by the nearest neighbors, $S$ is the average value of the manganese spin, and $\zeta(5 / 2)$ is the Riemann integral. 


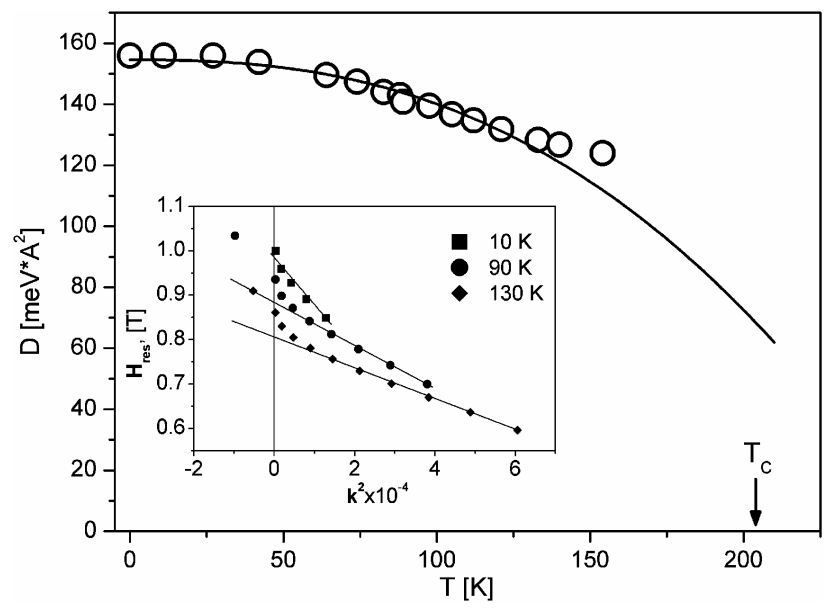

Fig. 2. The spin-wave stiffness coefficient $D$ vs. $T$ for annealed sample. The solid curve presents fitting of Eq. (6) to experimental points. The arrow points the Curie temperature position. The inset shows three dependences of the resonance field, $H_{\text {res }}$, versus the square of the spin wave vector $k^{2}$ at $T=10 \mathrm{~K}, 90 \mathrm{~K}, 130 \mathrm{~K}$. Note that the values of $k$ are normalized to the lattice constant and therefore are dimensionless.

$\bar{l}^{2}$ is defined by $\bar{l}^{2}=(S / 3 D)\left[\sum l^{n+2} J(\boldsymbol{l})\right]$, which gives information on the range of the exchange interaction. The solid curves in Fig. 2 are fittings of Eq. (5) to the experimental data being in good agreement with them for temperatures up to $165 \mathrm{~K}$, which is quite close to the Curie point, $T_{\mathrm{C}}=205 \mathrm{~K}$. The fitted values of $\bar{l}^{2}$ give $\sqrt{\bar{l}^{2}}=5.4 a_{0}$, where $a_{0}$ is here the distance to the nearest neighbors $(3.907 \AA)$, and indicate that the exchange interaction extends significantly beyond nearest neighbors. For $T>165 \mathrm{~K}$ the resonance peaks becomes unresolved not allowing the D evaluation. However, it should be noted that the experimental $D(T)$ dependence departures from theoretical curve for $T>150 \mathrm{~K}$, when the SWR spectrum begins to show the splitting. It is known for other manganites $[16,39]$ that, at temperatures close to $T_{\mathrm{C}}$, their $D(T)$ dependences have rather power-law behavior, and appearing to collapse as $T \rightarrow T_{\mathrm{C}}$. In $\mathrm{La}_{0.7} \mathrm{Mn}_{1.3} \mathrm{O}_{3-\delta}$ the deviation in opposite direction could be explained by decreasing of $\bar{l}^{2}$, which mean that itinerant $e_{\mathrm{g}}$ electrons become localized and it is consistent with charge ordering and stripe formation of $\mathrm{Mn}$ ions.

The spin-wave stiffness at $T=0 \mathrm{~K}, D(0)$, is related to intrinsic property of magnets and it seems to be of some interest to compare these values for different manganites known from the literature. Figure 3 shows the $D(0)$ as a function of the doping level $x$ evaluated in different manganites with the aid of both: inelastic neutron scattering $[10-12,15,16,23,25,28]$ and microwave techniques $[39,43]$. The $D(0)$ values obtained using the SWR are in good agreement with those measured in other ferromagnetic manganites. There are no compound that 


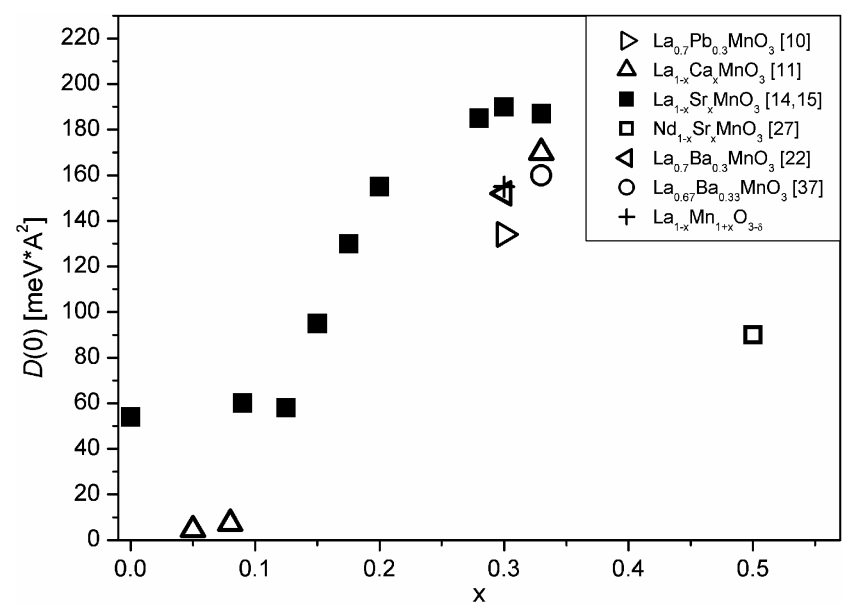

Fig. 3. The spin-wave stiffness coefficient, $D(0, x)$, evaluated from both: inelastic neutron scattering and microwave techniques, as a function of the doping level $x$.

was studied by two techniques simultaneously, however $D(0)$ values are very similar for $\mathrm{La}_{0.7} \mathrm{Ba}_{0.3} \mathrm{O}_{3}$ studied by the INS technique and $\mathrm{La}_{0.67} \mathrm{Ba}_{0.33} \mathrm{O}_{3}$ studied by the SWR.

\section{Conclusions}

The review of experimental studies of manganites shows that the magnons dominate the spectrum of magnetic excitations. For small wave vectors at low temperature the dispersion relation is quadratic as in a Heisenberg ferromagnet. In the case of thin films, inelastic neutron scattering experiments can be successfully replaced by microwave technique for studying spin dynamics. Besides bulk magnetic properties, the spin wave resonance spectrum allows one to make deep insight into surface effects, like excitation of surface spin waves or stripe formation as it was shown in an example of $\mathrm{La}_{0.7} \mathrm{Mn}_{1.3} \mathrm{O}_{3-\delta}$ film.

\section{Acknowledgment}

This work was partially supported by the State Committee for Scientific Research under contract 1 P03B 02526.

\section{References}

[1] Y. Tokura, N. Nagaosa, Science 288, 462 (2000).

[2] J.B. Goodenough, Aust. J. Phys. 52, 155 (1999).

[3] J.M.D. Coey, M. Viret, S. von Molnar, Adv. Phys. 48, 167 (1999). 
[4] E. Dagotto, T. Hotta, A. Moreo, Phys. Rep. 344, 1 (2001).

[5] S.P.P. Parkin, Ann. Rev. Mater. Sci. 25, 357 (1995).

[6] G. Zhao, K. Conder, H. Keller, K.A. Mueller, Nature 380, 676 (1996).

[7] Y. Moritomo, A. Asamitsu, H. Kuwahara, Y. Tokura, Nature 380, 141 (1996).

[8] H.L. Ju, C. Kwon, Q. Li, L. Green, T. Venkatesan, Appl. Phys. Lett. 65, 2108 (1994).

[9] N. Furukawa, J. Phys. Soc. Jpn. 65, 1174 (1996).

[10] T.G. Perring, G. Aeppli, S.M. Hayden, S.A. Carter, J.P. Remeika, S.-W. Cheong, Phys. Rev. Lett. 77, 711 (1996).

[11] F. Moussa, M. Hennion, J. Rodriguez-Carvajal, H. Moudden, L. Pinsard, A. Revcolevschi, Phys. Rev. B 54, 15149 (1996).

[12] J.W. Lynn, R.W. Erwin, J.A. Borchers, Q. Huang, A. Santoro, J.L. Peng, Z.Y. Li, Phys. Rev. Lett. 76, 4046 (1996).

[13] M.C. Martin, G. Shirane, Y. Endoh, K. Hirota, Y. Moritomo, Y. Tokura, Phys. Rev. B 53, 14285 (1996).

[14] L. Vasiliu-Doloc, J.W. Lynn, A.H. Moudden, A.M. de Leon-Guevara, A. Revcolevschi, J. Appl. Phys. 81, 5491 (1997).

[15] A.H. Moudden, L. Visiliu-Doloc, L. Pinsard, A. Revcolevschi, Physica B 241, 276 (1998).

[16] L. Vasiliu-Doloc, J.W. Lynn, A.H. Moudden, A.M. de Leon-Guevara, A. Revcolevschi, Phys. Rev. B 58, 14913 (1998).

[17] L. Vasiliu-Doloc, J.W. Lynn, Y.M. Mukovskii, A.A. Arsenov, D.A. Shulyatev, J. Appl. Phys. 83, 7342 (1998).

[18] H.Y. Hwang, P. Dai, S.-W. Cheong, G. Aeppli, D.A. Tennant, H.A. Mook, Phys. Rev. Lett. 80, 1316 (1998).

[19] J.A. Fernandez-Baca, P. Dai, H.Y. Hwang, S.-W. Cheong, C. Kloc, Phys. Rev. Lett. 80, 4012 (1998).

[20] J.J. Rhyne, H. Kaiser, H. Luo, G. Xiao, M.L. Gardel, J. Appl. Phys. 83, 7339 (1998).

[21] Pengcheng Dai, H.Y. Hwang, Zhang Jiandi, J.A. Fernandez-Baca, S.-W. Cheong, C. Kloc, Y. Tomioka, Y. Tokura, Phys. Rev. B 61, 9553 (2000).

[22] G. Jackeli, N.B. Perkins, N.M. Plakida, Phys. Rev. B 64, 092403 (2001).

[23] T. Chatterji, L.P. Regnault, W. Schmidt, Phys. Rev. B 66, 214408 (2002).

[24] J.A. Fernandez-Baca, Pengcheng Dai, H. Kawano-Furukawa, H. Yoshizawa, E.W. Plummer, S. Katano, Y. Tomioka, Y. Tokura, Phys. Rev. B 66, 054434 (2002).

[25] F. Moussa, M. Hennion, P. Kober, F. Wang, J. Carvajal-Rodriguez, P. Reutler, Y.M. Mukovskii, D. Shulyatev, J. Magn. Magn. Mater. 258-259, 259 (2003).

[26] J.W. Lynn, J. Supercond. 13, 263 (2000).

[27] G. Khaliullin, R. Kilian, J. Phys., Condens. Matter 11, 9757 (1999).

[28] R. Kajimoto, H. Yoshizawa, H. Kawano-Furukawa, H. Kuwahara, Y. Tomioka, Y. Tokura, J. Magn. Magn. Mater. 226-230, 892 (2001). 
[29] A.J. Millis, B.I. Shraiman, R. Mueller, Phys. Rev. Lett. 77, 175 (1996).

[30] H. Röder, J. Zang, A.R. Bishop, Phys. Rev. Lett. 76, 1356 (1996).

[31] A.M. Portis, Appl. Phys. Lett. 2, 69 (1963).

[32] C. Kittel, Phys. Rev. 110, 1295 (1958).

[33] R.F. Soohoo, Phys. Rev. 131, 594 (1963).

[34] H. Puszkarski, Acta Phys. Pol. A 38, 217 (1970).

[35] H. Puszkarski, Acta Phys. Pol. A 38, 899 (1970).

[36] H. Puszkarski, Prog. Surf. Sci. 9, 191 (1979).

[37] J. Spalek, Acta Phys. Pol. A 43, 635 (1973).

[38] I. Tamm, Physikalische Zeitschrift der Sowietunion 1, 733 (1932).

[39] S.E. Lofland, S.M. Bhagat, C. Kwon, M.C. Robson, R.P. Sharma, R. Ramesh, T. Venkatesan, Phys. Lett. A 209, 246 (1995).

[40] J. Yin, Y.X. Sui, J.H. Du, Y.X. Zhang, X.Y. Liu, Z.G. Liu, Phys. Status Solidi A 174, 499 (1998).

[41] V. Dyakonov, A. Prohorov, V. Shapovalov, S. Khartsev, V. Krivoruchko, V. Mihailov, V. Pashchenko, E. Zubov, P. Aleshkevych, K. Dyakonov, S. Piechota, H. Szymczak, Phys. Lett. A 268, 202 (2000).

[42] V. Dyakonov, A. Prohorov, V. Shapovalov, V. Krivoruchko, V. Pashchenko, E. Zubov, V. Mihailov, P. Aleshkevych, M. Berkowski, S. Piechota, H. Szymczak, J. Phys., Condens. Matter 13, 4049 (2001).

[43] P. Aleshkevych, J. Lindner, K. Baberschke, V. Dyakonov, H. Szymczak, R. Szymczak, Phys. Status Solidi A 196, 93 (2003).

[44] V.N. Krivoruchko, S.I. Khartsev, Low Temp. Phys. 24, 803 (1998).

[45] V.N. Krivoruchko, S.I. Khartsev, A.D. Prokhorov, V.I. Kamenev, R. Szymczak, M. Baran, M. Berkowski, J. Magn. Magn. Mater. 207, 168 (1999).

[46] H. Puszkarski, M. Krawczyk, Phys. Lett. A 282, 106 (2001).

[47] S. Mori, C.H. Chen, S.-W. Cheong, Nature 392, 473 (1998).

[48] C.H. Chen, S.-W. Cheong, H.Y. Hwang, J. Appl. Phys. 81, 4326 (1997).

[49] S. Yunoki, T. Hotta, E. Dagotto, Phys. Rev. Lett. 84, 3714 (2000).

[50] R. Klingeler, J. Geck, R. Gross, L. Pinsard-Gaudart, A. Revcolevschi, S. Uhlenbruck, B. Büchner, Phys. Rev. B 65, 174404 (2002).

[51] J.C. Loudon, N.D. Mathur, P.A. Midgley, Nature 420, 797 (2002).

[52] D.C. Mattis, The Theory of Magnetism, Springer-Verlag, Heidelberg 1981. 